et al. have shown that rapid hand BMD loss occurred in patients with undifferentiated arthritis who were later diagnosed with RA, and not in those later diagnosed with other conditions. BMD of the femoral neck and spine did not distinguish between patients with RA and those with other conditions.

Their prospective, longitudinal, UK study enrolled 100 consecutive patients with undifferentiated arthritis of <12 months' duration. None had previously taken diseasemodifying antirheumatic drugs or prednisolone. BMD of the femoral neck, spine, and whole hands were measured in 74 patients: during 12 months of follow-up, 13 patients were diagnosed with RA, 19 were diagnosed with a non-RA inflammatory disorder, and 42 were diagnosed with a noninflammatory joint disorder. The authors found that rheumatoid-factor status and elevated C-reactive protein levels were independent predictors of hand BMD loss, which confirms their importance as markers of RA disease activity and severity. Hand BMD was not reduced by corticosteroid use.

The authors measured mean global BMD of both hands, by dual-energy X-ray absorbtiometry. They conclude that this technique is a sensitive tool for measuring hand bone loss in early RA that could identify patients at high risk of progressive disease.

Original article Haugeberg G et al. (2006) Hand bone loss in early undifferentiated arthritis: evaluating bone mineral density loss before the development of rheumatoid arthritis. Ann Rheum Dis 65: 736-740

\section{Botulinum A toxin can give sustained relief from chronic low-back pain}

Many treatments for low-back pain exist, but results are often poor and ineffective. Moreeffective treatments are desired. Ney et al. demonstrate that successive botulinum $A$ toxin (BTX) injections are well tolerated and can provide relief for chronic low-back pain. Importantly, $16 \%$ of their BTX-treated patients experienced sustained pain relief (duration $>4$ months) from a single injection, without re-treatment. Ney and colleagues found BTX injection improved lumbosacral radicular pain, as well as low-back pain. Initial response to BTX predicted a continued beneficial response to re-treatment.
Their open-label, prospective study enrolled 18 women and 42 men aged 21-79 years with chronic low-back pain (mean duration 9 years) largely refractory to conventional treatment. Patients received four or five paraspinal BTX injections per affected side from $L 2$ to $S 1$, at a dose of 40-50 U per site (total dose $\leq 500 \mathrm{U}$ ). Pain and responses to treatment were assessed using a visual analog scale and two validated low-back-pain questionnaires. Over half of the patients evaluated at 3 weeks responded to treatment and maintained a response for $\geq 2$ months. Patients who initially responded were re-treated after 4 months if pain had returned. No weakness or gait instability was reported, and other adverse effects of treatment were mild and uncommon.

Both peripheral and central mechanisms are postulated in BTX-related pain relief. Ney et al. call for randomized, double-blind trials to confirm the therapeutic benefit of BTX, and for basic research to characterize its analgesic properties.

Original article Ney JP et al. (2006) Treatment of chronic low back pain with successive injections of botulinum toxin A over 6 months: a prospective trial of 60 patients. Clin J Pain 22: $363-369$

\section{Interleukin-6-receptor blockade increases adrenal androgen levels}

Both tumor necrosis factor (TNF) and interleukin 6 (IL-6) are known to affect the adrenal gland, but the bulk of the existing literature has focused on TNF. For instance, although TNF is known to inhibit adrenal steroidogenesis in patients with rheumatoid arthritis, it is unknown whether IL-6 has a similar role. In order to clarify this issue, researchers in Germany and Japan have examined the effect of monoclonal antibodies to the IL-6 receptor (MRA) on steroidogenesis in a double-blind, randomized, placebo-controlled study.

Patients with rheumatoid arthritis treated with prednisolone were assigned to receive either MRA $(n=16)$ or placebo $(n=13)$ over the 12 week study period. Patients in the MRA group showed marked improvements in clinical signs of inflammation compared with patients in the placebo group. The serum levels of eight hormones were measured at baseline and every 2 weeks thereafter; no changes were seen within 